Proceedings of the 6th Polish Symposium of Physics in Economy and Social Sciences (FENS2012), Gdańsk, Poland

\title{
The Efficiency of Polish Stock Market: Ordinal Patterns Approach
}

\author{
G. GRAFF* AND A. KaCZKOWsKA
}

Faculty of Applied Physics and Mathematics, Gdańsk University of Technology, Gdańsk, Poland

\begin{abstract}
Zunino et al. analyzed the problem of discrimination of developed and emergent markets by the use of ordinal patterns methods: number of forbidden patterns and ordinal pattern probability distribution as a basis for entropy and statistical measure of complexity. In this paper we apply the same methodology for the analysis of Polish stock market (index WIG). The results indicate that Polish market belongs neither to developed, nor to emergent groups of markets. It represents hybrid market, the third group distinguished in the paper by Zunino et al.
\end{abstract}

DOI: $10.12693 /$ APhysPolA.123.526

PACS: 89.65.Gh, 89.70.Cf, 89.75.Kd

\section{Introduction}

The Polish market is recently a subject of growing interest [1-4]. In this paper we deal with the problem of its efficiency in comparison to other world's markets. The notion of efficiency of a market is related to its unpredictability, i.e. an ideal efficient market could be characterized by the fact that prices are not at all predictable but random, and thus there is no clear investment pattern. The developed markets are highly efficient in the opposition to the emerging ones, which are more deterministic and thus more predictable.

Recent investigations of Zunino et al. [5, 6] have shown that ordinal patterns methods are very useful base to build various mathematical devices that classify markets according to their efficiency. In $[5,6]$ the authors analyzed 32 developed and emergent markets, using number of forbidden patterns, permutation entropy and statistical complexity measure. In this paper we apply the same tools to study the efficiency of Polish stock market, not mentioned in $[5,6]$.

\section{Methods}

\subsection{Ordinal patterns}

Ten years ago Bandt and Pompe introduced a new approach to time series analysis [7]. Their main idea was to assign to data some symbols and to investigate order relations between them. This kind of symbolic analysis turned out to be very fruitful, as it is appropriate for nonstationary data, it does not demand establishing probability density function and is robust against noise $[8,9]$.

For a given series $\left\{x_{t}\right\}_{t=1}^{N}$ a sliding window of length $D$, $x_{n+1}, \ldots, x_{n+D}(0 \leq n \leq N-D)$ is considered and denoted as $x_{n+1}^{n+D}$. By ordering elements of the window we

*corresponding author; e-mail: graff@mif.pg.gda.pl obtain

$$
x_{n+\pi_{1}}<x_{n+\pi_{2}}<\ldots<x_{n+\pi_{D}}
$$

which defines an ordinal pattern of $x_{n+1}^{n+D}$ as the permutation $\pi=\left\langle\pi_{1}, \pi_{2}, \ldots, \pi_{D}\right\rangle$. For example, if $D=4$ and $x_{n+1}=3, x_{n+2}=-5, x_{n+3}=0, x_{n+4}=1$ then the ordinal pattern of this window is given by the permutation $\pi=\langle 2,3,4,1\rangle$. The problem with defining the permutation patterns for series with equal values could be solved in many ways. In our approach we apply the convention that if $x_{i}=x_{j}$, then we set $x_{i}<x_{j}$ for $i<j$ (see also [8]).

\subsection{Forbidden patterns}

There are $D$ ! order patterns of length $D$ and for a given series some of them may not appear. They are called forbidden patterns. The number of forbidden patterns (abbreviated as NFP) is a good instrument to discriminate deterministic series from random ones. For random time series number of forbidden patterns tends to zero, when the length of the series is long enough, while for deterministic systems, even very noisy, some forbidden patterns could persist for a long time.

The methods based on forbidden patterns analysis have many advantages, among which there are their simplicity and computational efficiency. The value of $D$ should be chosen so that $N \gg D$ ! to allow every possible pattern of length $D$ to appear in the series of length $N$.

\subsection{Normalized permutation entropy}

We will use ordinal pattern probability distribution as a basis for entropy and statistical measure of complexity. The relative frequencies are defined as

$$
\begin{aligned}
& p\left(\pi_{i}\right)= \\
& \frac{\mid\left\{n: 0 \leq n \leq N-D, x_{n+1}^{n+D} \text { is of the type } \pi_{i}\right\} \mid}{N-D+1},
\end{aligned}
$$

and then the ordinal pattern probability distribution is given by

$$
P=\left\{p\left(\pi_{i}\right): i=1,2, \ldots, D !\right\} .
$$

Let us recall that the classical Shannon entropy, for a given probability distribution $P$, is defined as: 


$$
S[P]=-\sum_{i} p_{i} \ln p_{i} .
$$

For probability distribution (1) we define normalized permutation entropy as

$$
\mathcal{H}_{S}(P)=S[P] / S_{\max }=\left[-\sum_{i=1}^{D !} p\left(\pi_{i}\right) \ln p\left(\pi_{i}\right)\right] / S_{\max },
$$

where $S_{\max }=\ln D !\left(0 \leq \mathcal{H}_{S} \leq 1\right)$.

\subsection{Complexity-entropy causality plane}

We will use statistical complexity measure introduced by Lamberti et al. [10] since it is a useful tool to discriminate different degrees of periodicity and chaos. To define the statistical complexity measure, we use uniform distribution $P_{e}=\left\{\frac{1}{N}, \ldots, \frac{1}{N}\right\}$ and introduce the JensenShannon divergence

$$
J\left[P, P_{e}\right]=S\left[\frac{P+P_{e}}{2}\right]-\frac{S[P]}{2}-\frac{S\left[P_{e}\right]}{2},
$$

where $S$ denotes the Shannon entropy. We determine the so-called disequilibrium

$$
\mathcal{Q}_{J}\left[P, P_{e}\right]=\mathcal{Q}_{0} J\left[P, P_{e}\right],
$$

where $\mathcal{Q}_{0}$ is a normalization constant, equal to the inverse of the maximal possible value of $J\left[P, P_{e}\right]$ (this number is obtained when one of the components of $P$ is equal to one and the other are equal to zero).

Then, the statistical complexity measure is defined as

$$
\mathcal{C}_{J S}[P]=\mathcal{Q}_{J}\left[P, P_{e}\right] \mathcal{H}_{S}[P] .
$$

In the present work normalized permutation entropy given in (2) and statistical complexity measure defined in (3) are evaluated by a use of ordinal patterns probability distribution (1).

Another tool that will be used is the complexity-entropy causality plane, introduced in [11] as the representation space obtained with normalized permutation entropy in the horizontal axis and statistical complexity measure in the vertical one. It has been recently shown that complexity-entropy causality plane is an effective tool for distinguishing the stage of stock and commodity markets development $[2,12]$.

\section{Results}

In this section we apply the methods described in Sect. 2 for Polish stock market. Following [5] we use daily data of equity index WIG from January 2th, 1995 to July 23rd, 2007 (3138 observations - only trading days) and $D \in\{4,5,6\}$. The data were extracted from the website www.wyborcza.biz/Gieldy.

It was shown in [1] that both number of forbidden patterns and normalized permutation entropy could be successfully used to assess the degree of stock market development. Developed markets have lower number of forbidden patterns and higher normalized permutation entropy in opposition to emergent stock markets. The results obtained for the number of forbidden patterns and normalized permutation entropy for Polish market are presented in Table, where the indices for Poland are compared with averages for 18 developed and 14 emerging markets. Let us mention that number of forbidden patterns gives zero value for $D<6$. These results suggest that Polish stock market is characterized by the middle efficiency, and thus it is a hybrid type of market.

\section{TABLE}

NFP and NPE for Poland and other countries. The averages for developed $\left(A v_{d e v}\right)$ and emerging $\left(A v_{e m}\right)$ markets are taken from [5] and given in form average \pm SD (SD standard deviation).

\begin{tabular}{c|c|c|c|c}
\hline \hline & NFP & \multicolumn{3}{|c}{ NPE } \\
\cline { 3 - 5 } & $D=6$ & $D=4$ & $D=5$ & $D=6$ \\
\hline Poland & 166 & 0.919 & 0.888 & 0.851 \\
Av $_{\text {dev }}$ & $141 \pm 21$ & $0.929 \pm 0.01$ & $0.900 \pm 0.01$ & $0.865 \pm 0.01$ \\
Av $_{\text {em }}$ & $184 \pm 14$ & $0.906 \pm 0.01$ & $0.880 \pm 0.03$ & $0.835 \pm 0.01$
\end{tabular}

There exists the negative relationship between the degree of country development and number of forbidden patterns (for $D=6$ ) [5], where the development is measured by the Gross National Income per capita (data from year 2006, in US\$, taken from World Bank website). On the other hand, for normalized permutation entropy, its relationship with Gross National Income is positive. In Fig. 1 Polish market is situated among other world's markets considered in [5]. Although close to the area of emergent markets it is a little shifted to the region of developed ones.

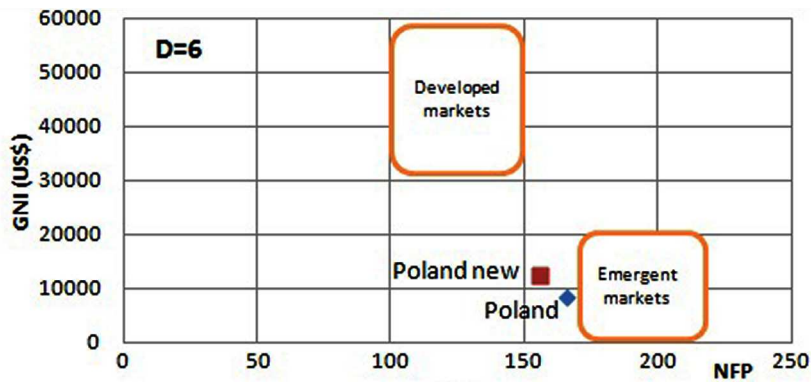

Fig. 1. The gross national income (GNI) per capita as a function of NFP with $D=6$.

The strong argument that supports the hypothesis of hybridity of Polish market is given by the use of complexity-entropy causality plane representation (see Fig. 2). Here, the data for different countries are grouped near the line from the left top to the right bottom. On the left, there are emergent markets (higher statistical complexity measure, lower normalized permutation entropy) and on the right - developed ones (lower statistical complexity measure, higher normalized permutation entropy) [6]. There is a group of countries that are placed in the middle of complexity-entropy causality plane. Thus, Zunino et al. identified the third kind of market: hybrid. It takes intermediate values of statistical complexity measure and normalized permutation entropy 
and covers ten of the countries investigated in [6] (Argentina, Austria, Brazil, Canada, China, Greece, Hong Kong, Singapore, Taiwan and Turkey - five emergent and five developed due to MSCI criterion). The position of Poland in complexity-entropy causality plane shows that it can be regarded as a country with the hybrid market, because it also takes intermediate values of statistical complexity measure and normalized permutation entropy (Fig. 2, index POLAND).

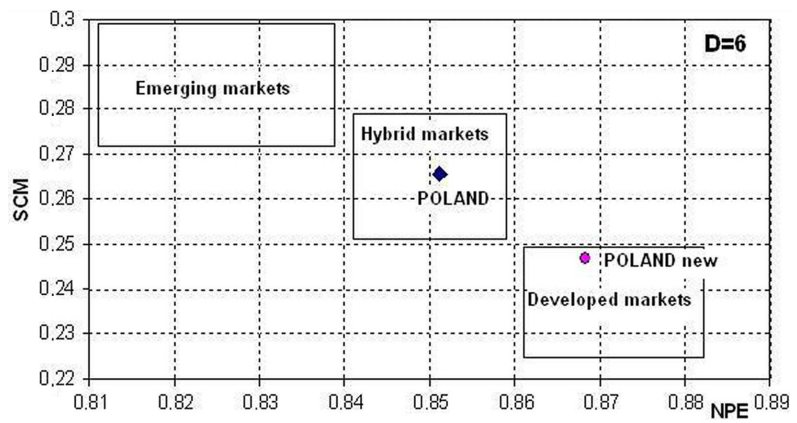

Fig. 2. Complexity-entropy causality plane for Polish market (old and latest indices) with the division to developed, hybrid and emergent markets. NPE - normalized permutation entropy, SCM - statistical complexity measure.

In order to examine the evolution of Polish market we analyzed the latest observations of WIG index (3137 trading days from February 2nd, 2000 to September 28th, 2012), obtaining for $D=6$ the decrease in NFP and increase in normalized permutation entropy (NPE): $\mathrm{NFP}=156$ and NPE $=0.869$. Furthermore, if we place these results in Fig. 2 (index POLAND new) it turns out that Polish market is situated in the group of developed ones. The similar observation could be made by the analysis of Fig. 1, in which the index based on the recent data for Polish market (POLAND new) is closer to the area of developed countries. Of course, the world economy is also developing, so there must be some shift for other countries appearing in Fig. 2, and thus it is difficult to determine the present position of Polish market. Nevertheless, we can draw a conclusion that current Polish economy is in the process of increasing its efficiency.

\section{References}

[1] W. Bachnik, P. Chomiuk, Sz. Faltynowicz, M. Gawin, W. Gorajek, J. Kedzierski, K. Kosk, A. Kucharczyk, P. Leszczynski, R. Podsiadlo, D. Makowiec, Acta Phys. Pol. B 36, 2435 (2005).

[2] L. Czarnecki, D. Grech, G. Pamuła, Physica A 387, 6801 (2008).

[3] P. Gnaciński, D. Makowiec, Physica A 344, 322 (2004).

[4] D. Grech, G. Pamuła, Physica A 387, 4299 (2008).

[5] L. Zunino, M. Zanin, B.M. Tabake, D.G. Pérez, O.A. Rosso, Physica A 388, 2854 (2009).

[6] L. Zunino, M. Zanin, B.M. Tabake, D.G. Pérez, O.A. Rosso, Physica A 389, 1891 (2010).

[7] C. Bandt, B. Pompe, Phys. Rev. Lett. 88, 174102 (2002).

[8] J.M. Amigo, Permutation Complexity in Dynamical Systems. Ordinal Patterns, Permutation Entropy and All That, Springer-Verlag, Berlin 2010.

[9] M. Zanin, L. Zunino, O.A. Rosso, D. Papo, Entropy 14, 1553 (2012).

[10] P.W. Lamberti, M.T. Martin, A. Plastino, O.A. Rosso, Physica A 334, 119 (2004).

[11] O.A. Rosso, H.A. Larrondo, M.T. Martin, A. Plastino, M.A. Fuentes, Phys. Rev. Lett. 99, 154102 (2007).

[12] L. Zunino, A. Fernandez Bariviera, M. Belen Guercio, L.B. Martinez, O.A. Rosso, Physica A 391, 4342 (2012). 